\title{
Review of: "A meta-analysis of the effects of glucagon-like-peptide 1 receptor agonist (GLP1-RA) in nonalcoholic fatty liver disease (NAFLD) with type 2 diabetes (T2D)"
}

\author{
Margarita Papatheodoridi, George Papatheodoridis ${ }^{1}$ \\ 1 University of Athens
}

Potential competing interests: The author(s) declared that no potential competing interests exist.

To date, there is no approved treatment with clear benefit for patients of non-alcoholic fatty liver disease (NAFLD), which is an increasing cause of end-stage liver disease and hepatocellular carcinoma. The prevalence of NAFLD is extremely high in patients with type 2 diabetes (T2D), who often progress to advanced stages of NALFD. Thus, an effective treatment for NAFLD in patients with T2D will be extremely useful. Given that the two diseases not only co-exist but share the same pathophysiological mechanisms like insulin resistance, obesity and particularly central obesity, metabolic syndrome, splanchnic fat accumulation and deranged adipokines levels, it is reasonable to assume that the same agents may be effective for both NAFLD and T2D. Glucagon-like-peptide 1 receptor agonists (GLP1-RAs) represent a class of agents that has been evaluated for both NAFLD and T2D. Although GLP1-RAs have been approved for the treatment of T2D, no final conclusion has been made about their efficacy in NAFLD, while at least one representative of these agents, semaglutide, is currently under investigation in phase III clinical trials for the treatment of patients with NAFLD regardless of the presence of T2D.

Ghosal et al. recently published a meta-analysis of 8 randomized controlled trials on GLP1-RAs in patients with NAFLD and T2D. According to their results, such patients who were treated with a GLP1-RA $(n=297)$, compared to controls $(n=318$, treated with insulin in three, metformin in one, standard of care in two and placebo in two trials) achieved lower alanine aminotransferase (ALT), aspartate aminotransferase (AST), gamma-glutamyl transpeptidase (GGT) and glucosylated haemoglobin serum levels as well as improved liver fat content, body weight and histological lesions.

The results of the above meta-analysis are interesting, but at the same time several concerns and uncertainties arise. The main limitation and uncertainty are related to the great heterogeneity in key characteristics of the included studies. First, various GLP1-RAs were used in the main arms (dulaglutide in one, exenatide in three, liraglutide in three and semaglutide in one study), when it is uncertain whether different GLP1-RAs have the same effect on NAFLD. Second, treatment durations of the included studies ranged widely from 12 to 72 weeks. Although there is not enough data on this issue, treatment duration probably plays an important role, especially when assessing the effects of the treatment on liver histology. Third, there was great heterogeneity in the control arm which included placebo ( 2 studies), metformin ( 1 
study), insulin (3 studies) or standard of card without further definition ( 2 studies). Although none of the above anti-diabetic agents has been shown to have a clear effect on NAFLD, nobody can exclude that a control arm with any antidiabetic agent may differ than placebo, as it is unclear whether better control of diabetes is without any effect on NAFLD.

With regards to the liver-related outcome measurements, liver fat content was lower in the GLP1-RAs arm in two studies only, which used MR-PDFF. Similarly, histological improvement was also based on a metanalysis of two studies only, which also had different study durations (48 and 72 weeks). Consequently, the reliability of these findings coming of only two studies may be questioned.

On a different note, there are several other meta-analyses on the effects of GLP1-RAs on NAFLD patients usually with but also without T2D and all report similar findings from a small number of included studies. However, more data are required before the use of GLP1-RAs can be decided and included in the NAFLD guidelines. Although the authors state that “This meta-analysis indicates that GLP-1 RA is potentially a robust treatment strategy in patients with T2D and NAFLD" and that they "believe GLP1-RA will soon be a useful therapeutic option to manage T2DM patients with NAFLD", no clear recommendation can be made yet. Given the heterogeneity of such patients and the complexity of the assessment of treatment responses in NAFLD patients, the results of large phase III trials are required to clarify which GLP1-RA may be effective, at which dose and for how long should be given as well as which is going to be the anticipated benefit. Based on the existing data and the pathophysiologic rationale, most clinicians can accept that GLP1-RA may be selected for the treatment of T2D in patients with NAFLD as well, if GLP1-RA is one of the first choices for T2D management in those patients. 